\title{
Avaliação de Desempenho dos Modelos de Mudança Climática do IPCG-AR4 Quanto a Sazonalidade e os Padrões de Variabilidade Interanual da Precipitação Sobre o Nordeste do Brasil, Bacia do Prata e Amazônia
}

\author{
Cleiton da Silva Silveira*, Francisco de Assis de Souza Filho*, Yvonne Magdalena Campos Lázaro" \\ cleitonsilveira16@yahoo.com.br, assissouzafilho@gmail.com,yvonnecamp@gmail.com
}

Recebido: 29/10/11 - revisado: 20/11/11 - aceito: 30/11/12

\begin{abstract}
RESUMO
Os modelos globais do quarto relatório do Intergovernmental Panel on Climate Change (IPCC) são avaliados para o Nordeste Setentrional do Brasil (NEB), Bacia do Prata e Amazônia quanto à representação da precipitação para o periodo de 1901 a 1999. Essa avaliação é realizada utilizando-se os dados do Climatic Research Unit (CRU) e a reanálise 20th Century Reanalysis V2 do National Oceanic and Atmospheric Administration (NOAA). A avaliação é constituída por três etapas que analisam padrões de variação de diferentes escalas: sazonal, interanual e interdecadal. A avaliação sazonal é baseada em medidas de correlação e erro quadrático médio em relação à climatologia média das regiões. Enquanto as avaliações interanual e interdecadal são baseadas na transformada em ondoletas. A maioria dos modelos captura os padrões de variação sazonal e interanual, porém possui muita dificuldade de representar a variabilidade interdecadal. Os melhores membros do conjunto de simulações do IPCC-AR4 para o século XX segundo esses critérios foram: na região da Amazônia as rodadas do giss_model_e_r, no NEB o csiro.mk3.0.run2 e na Bacia da Prata as rodadas do modelo cccma_cgcm3_1.
\end{abstract}

Palavras-chave: Mudança climática, modelos. IPCC-AR4. América do Sul. Bacia do Prata. Amazônia.

\section{INTRODUÇÃO}

Diversos autores identificam aumento da temperatura planetária nas últimas décadas, combinado com modificações nos campos de precipitação, associando essas ocorrências às Mudanças Climáticas (CAMPO; NÉRIS, 2010; MARENGO; SOARES, 2005; MARENGO; VALVERDE, 2007; MILLY; DUNNE; VECCHIA, 2005; NÓBREGA et al., 2011; SALATI et al., 2008; TOMASELLA et al., 2009). Estas têm sido alvo de discussões e pesquisas científicas em todo mundo com vistas ao entendimento de sua ocorrência (IPCC, 2007a; NOBRE, 2005), assim como a identificação e a avaliação dos possíveis impactos ambientais, sociais e econômicos (IPCC, 2007b) e a elaboração de medidas que minimizem as conseqüências adversas das mesmas.

Entretanto, apesar do significativo desenvolvimento ocorrido nas últimas décadas acerca do conhecimento do clima, existem ainda diversas incertezas associadas aos vários sistemas de previsões ou projeções climáticas. Por exemplo, as parametrizações físicas, as condições iniciais e de fronteira

*Universidade Federal do Ceará adotadas pelo modelo. E ainda, o método numérico de resolução adotado pelos modelos, e a própria atmosfera, que é considerada um sistema caótico (LORENZ, 1965).

Quanto às questões relacionadas aos modelos globais de mudanças climáticas do Coupled Model Intercomparison Project Phase 3 (CMIP3) publicados pelo Intergovernmental Panel on Climate Change (IPCCAR4) pode se adicionar ainda a baixa resolução dos modelos que não é capaz de reproduzir alguns processos físicos e a trajetória desconhecida de emissões de gases de efeito estufa no futuro.

Dado o grande número de modelos e as projeções tão divergentes fornecidos pelos modelos globais do CMIP3, principalmente para o Nordeste do Brasil e Amazônia (LÁZARO, 2011; SILVEIRA; SOUZA FILHO, 2011), é imprescindível uma metodologia de avaliação que seja capaz de identificar os modelos que representam adequadamente as principais regiões da América do Sul para que possa ser dimensionada e tratada à incerteza associada às projeções do conjunto de modelos. Esta avaliação poderia ser vista por duas vertentes: a primeira consiste em identificar os melhores modelos e admitir que uma melhor representação do século XX levaria a uma representação mais coerente do futuro; já a 
segunda consiste em identificar os piores modelos e retira-los do conjunto para uma posterior análise estatística do futuro. Na América do Sul existem três regiões que notadamente possuem climas bastante distintos: o Nordeste do Brasil, a região amazônica e a Bacia da Prata.

O Nordeste do Brasil (NEB) apresenta clima semiárido com intensa variabilidade temporal e espacial de chuvas (ALBUQUERQUE et al., 2009; MOLION; BERNARDO, 2002) associada historicamente a significativos impactos sociais e econômicos sobre a região descritos em, por exemplo, Souza Filho e Moura (2006). Desta forma, torna-se relevante para o desenvolvimento de políticas públicas a identificação das alterações no clima nesta região.

Vários estudos vêm sendo realizados para retratar e tentar avaliar os impactos das mudanças climáticas na região amazônica (ALBUQUERQUE et al., 2009; MARENGO; VALVERDE, 2007; NOBRE; SELLER; SHUKL, 1991), isto pela grande contribuição que essa região exerce nos balaços hídricos e energéticos a nível global. Além desta, a região da Bacia do Prata é também objeto de estudo por se tratar da região onde se encontram as principais usinas fornecedora de energia elétrica do Brasil, e impactos climáticos nessa região poderiam ocasionar mudanças na oferta hídrica e energética do país.

O objetivo do presente estudo é desenvolver uma metodologia de avaliação da habilidade dos modelos do IPCC-AR4 de representar o regime de chuvas e aplicar esta ao Nordeste Setentrional do Brasil, Bacia do Prata e Amazônia.

\section{METODOLOGIA}

Esta seção apresenta as fontes de dados observacionais utilizados para as três regiões da América do Sul. Os modelos do IPCC-AR4 são classificados de acordo a sua capacidade de representar os padrões de precipitação nas escalas de tempo sazonal e plurianual no século XX para as três regiões de estudo. No caso da sazonalidade e da variabilidade plurianual, é feita uma comparação com base em alguns índices estatísticos para que possam ser definidos que modelos possuem melhor desempenho nas regiões de estudo.

A avaliação é constituída por três etapas que analisam padrões de variação de diferentes escalas: sazonal, interanual e interdecadal, conforme figura 1 e seção 2.4 .

\section{Região de Estudo}

Utilizaram-se três regiões para o estudo da variabilidade sazonal e plurianual da América do Sul. A primeira região corresponde a $0^{\circ}$ a $10^{\circ} \mathrm{S}$ de latitude e $33^{\circ} \mathrm{O}$ a $44^{\circ} \mathrm{O}$ de longitude sobre o Nordeste Setentrional Brasileiro assim como uma porção do Oceano Atlântico, delimitado pelo retângulo 1 conforme figura 2. A segunda região selecionada é delimitada pelo polígono 2 e vai de $3,5^{\circ} \mathrm{N}$ a $12^{\circ} \mathrm{S}$ de latitude e $75,50{ }^{\circ} \mathrm{O}$ a $49{ }^{\circ} \mathrm{O}$ de longitude sobre a Amazônia. A terceira região, delimitada pelo polígono 3 , vai de $36^{\circ} \mathrm{S}$ a $16,5^{\circ} \mathrm{S}$ de latitude e $64^{\circ} \mathrm{O}$ a $44^{\circ} \mathrm{O}$ de longitude, incluindo a Bacia da Prata, conforme figura 2 .

\section{Região Nordeste do Brasil}

A região NEB possui um ciclo anual bem definido, onde predominam dois períodos distintos: o período chuvoso e o período seco (ALBUQUERQUE et al., 2009). O período chuvoso distribui-se entre dezembro e julho e subdivide-se em: a) préestação chuvosa (dezembro e janeiro), durante a qual os principais sistemas causadores de chuva são a proximidade das frentes frias e os vórtices ciclônicos de ar superior; b) "quadra chuvosa" ou estação chuvosa propriamente dita (de fevereiro a maio), que tem a zona de convergência intertropical-ZCIT como principal sistema causador de chuva, impactando o setor norte do NEB, seguido de sistemas secundários tais como linhas de instabilidade, complexos convectivos de mesoescala e efeitos de brisa (UVO; NOBRE, 1987) e c) pós-estação chuvosa (junho e julho), em que os sistemas causadores de chuva mais importantes são as ondas de leste, que atuam principalmente sobre o leste do NEB, e os complexos convectivos de mesoescala. No segundo semestre, há um predomínio de altas pressões atmosféricas e uma quase total ausência de fenômenos atmosféricos causadores de chuva, caracterizando a estação seca. O NEB apresenta grande variabilidade de chuvas com regiões com precipitação de 500 $\mathrm{mm} /$ ano e outras com mais de $1400 \mathrm{~mm} /$ ano (SOUZA FILHO, 2003).

\section{Região da Amazônia}

A região amazônica, nos últimos anos, tem se destacado em diversos estudos pela grande contribuição no clima a nível global (ALBUQUERQUE et al., 2009). Essa contribuição se dá tanto por meio da sua extensa área florestal, que influencia bastante na circulação atmosférica global, como também de 


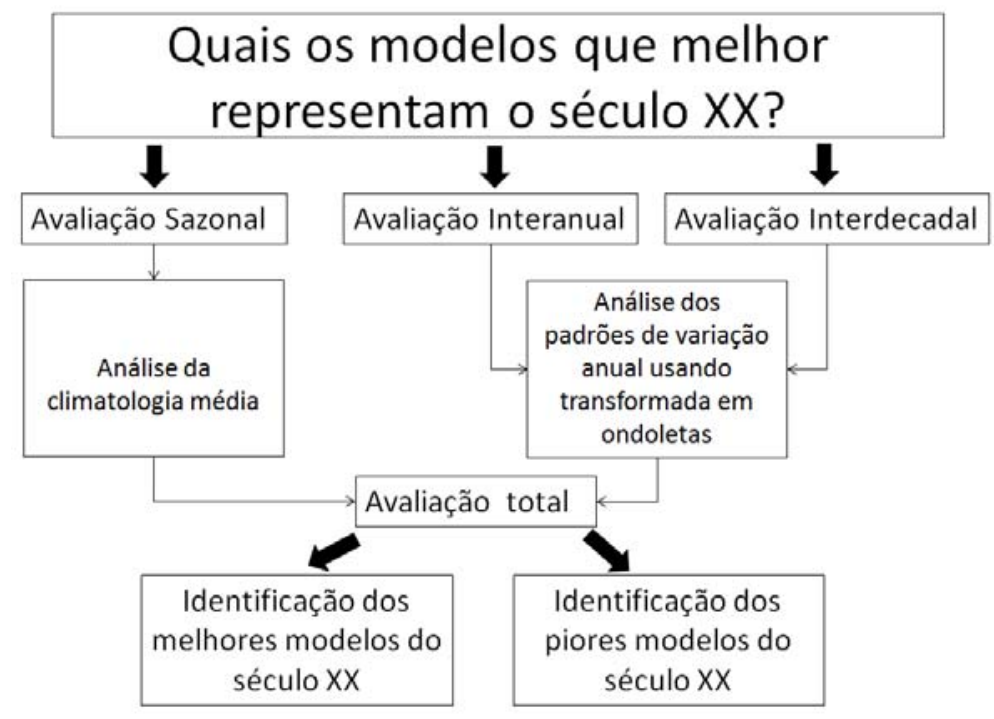

Figura 1 - Diagrama contendo aspectos da metodologia de avaliação dos modelos do CMIP3

sua contribuição hídrica com uma precipitação média de $2.300 \mathrm{~mm}$ por ano responsável por 220.000 $\mathrm{m}^{3} / \mathrm{s}$ de descarga média do rio Amazonas no oceano (18\% da descarga total de água doce nos oceanos), tornando-se assim facilmente uma região reguladora dos balaços hídricos e energéticos a nível global (ALBUQUERQUE et al., 2009).

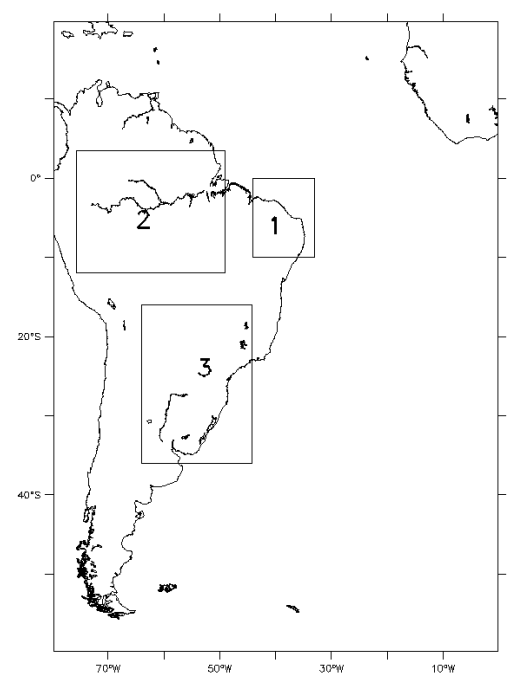

Figura 2 - América do Sul destacando para as regiões de estudo 1, 2 e 3. Estas representam, respectivamente, o Nordeste Setentrional brasileiro, Amazônia e Rio Prata
Em termos de sazonalidade o início da estação chuvosa no sul da Amazônia ocorre na primavera, ocorrendo os máximos de chuva no verão. Já para a região central que vai desde o oeste até a foz do Amazonas os máximos de chuva ocorrem no outono. Para o extremo norte a máxima de chuvas ocorre no período do inverno austral, época responsável pela estação seca nas regiões Central e Sul da Amazônia. Os trimestres mais secos na região Norte mudam progressivamente de setembro/outubro/ novembro no extremo norte, para agosto/ setembro/outubro, numa longa faixa latitudinal desde o oeste da região Nordeste; para julho/agosto/ setembro no vale da bacia Amazônica, sobretudo a oeste, e para junho/julho/agosto na parte sul.

\section{Região da Bacia do Prata}

A bacia da Prata é a segunda maior bacia hidrográfica do planeta. Os rios Paraná, Paraguai e Uruguai formam o rio da Prata. A bacia do rio Paraná apresenta o maior potencial hidrelétrico instalado do Brasil, além de trechos importantes para a navegação, pesca e outros usos relevantes.

$\mathrm{Na}$ bacia da Prata os principais sistemas causadores de chuvas são: à presença da Zona de Convergência do Atlântico Sul (ZCAS) e os Jatos de Baixos Níveis da América do Sul (SALLJ). O ciclo anual é bem definido, sendo as chuvas mais intensas na primavera (setembro-dezembro) e no outono (março-junho) (TEXEIRA; SATYAMURTY, 2007). 
Tabela 1 - Modelos globais do IPCC-AR4 utilizados no experimento

\begin{tabular}{|c|c|c|c|}
\hline Designação do Modelo & Instituição ou Agência; País & Topo do modelo / Resolução & $\begin{array}{l}\text { Rodadas } \\
\text { disponíveis }\end{array}$ \\
\hline BCCR-BCM2 & Bjerknes Centre for Climate Research, Universidade de Bergen; Noruega & $10 \mathrm{hPa} / \mathrm{T} 63\left(1.9^{\circ} \times 1.9^{\circ}\right) \mathrm{L} 31$ & run1 \\
\hline CCCMA-CGCM3 1-T47 & Canadian Centre for Climate Modelling and Analysis; Canadá & $1 \mathrm{hPa} / \mathrm{T} 47\left(\sim 2.8^{\circ} \times 2.8^{\circ}\right) \mathrm{L} 31$ & run1 a run5 \\
\hline CCCMA-CGCM3 1-T63 & Canadian Centre for Climate Modelling and Analysis; Canadá & $1 \mathrm{hPa} / \mathrm{T} 63\left(\sim 1.9^{\circ} \times 1.9^{\circ}\right) \mathrm{L} 31$ & run1 \\
\hline CNRM-CM3 & Centre National de RecherchesMeteorologiques, Meteo France; França & $0.05 \mathrm{hPa} / \mathrm{T} 63\left(\sim 1.9^{\circ} \times 1.9^{\circ}\right) \mathrm{L} 45$ & run1 \\
\hline CSIRO-MK3 & CSIRO; Austrália & $4.5 \mathrm{hPa} / \mathrm{T} 63\left(\sim 1.9^{\circ} \times 1.9^{\circ}\right) \mathrm{L} 18$ & run1 a run3 \\
\hline CSIRO-MK3.5 & CSIRO; Austrália & $4.5 \mathrm{hPa} / \mathrm{T} 63\left(\sim 1.9^{\circ} \times 1.9^{\circ}\right) \mathrm{L} 18$ & run1 a run3 \\
\hline GFDL-CM2.0 & Geophysical Fluid Dynamics Laboratory, NOAA; Estados Unidos & $3 \mathrm{hPa} / 2.0^{\circ} \times 2.5^{\circ} \mathrm{L} 24$ & run1 a run3 \\
\hline GFDL-CM2.1 & Geophysical Fluid Dynamics Laboratory, NOAA; Estados Unidos & $3 \mathrm{hPa} / 2.0^{\circ} \times 2.5^{\circ} \mathrm{L} 24$ & run1 e run2 \\
\hline INCM-CM3.0 & Institute of Numerical Mathematics, Russian Academy of Science; Rússia & $10 \mathrm{hPa} / 4^{\circ} \times 5^{\circ} \mathrm{L} 21$ & run1 \\
\hline INGV-SXG2005 & National Institute of Geophysics and Volcanology; Itália & $10 \mathrm{hPa} / \mathrm{T} 106\left(1,125^{\circ} \mathrm{x} 1,125^{\circ}\right)$ & run1 \\
\hline IPSL-CM4 & Institut Pierre Simon Laplace (IPSL); França & $4 \mathrm{hPa} / 2.5^{\circ} \times 3.75^{\circ} \mathrm{L} 19$ & run1 \\
\hline LASG-FGOALS-G1.0 & $\begin{array}{l}\text { LASG, Institute of Atmospheric Physics, Chinese Academy of Sciemces, P.O. Box 9804, Beijing } \\
\text { 100029; China }\end{array}$ & $2.2 \mathrm{hPa} / \mathrm{T} 42\left(\sim 2.8 \times 2.8^{\circ}\right) \mathrm{L} 26$ & run1 a run3 \\
\hline MPI-ECHAM5 & Max Planck Institute for Meteorology; Alemanha & $10 \mathrm{hPa} / \mathrm{T} 63\left(\sim 1.9^{\circ} \times 1.9^{\circ}\right) \mathrm{L} 31$ & run1 a run4 \\
\hline MRI-CGCM2.3.2 & Meteorological Research Institute, Japan Meteorological Agency; Japão & $0.4 \mathrm{hPa} / \mathrm{T} 42\left(\sim 2.8^{\circ} \times 2.8^{\circ}\right) \mathrm{L} 30$ & run1 a run4 \\
\hline NASA-GISS-AOM & Nasa Goddard Institute for Space Studies (NASA/GISS); Estados Unidos & $10 \mathrm{hPa} / 3^{\circ} \times 4^{\circ} \mathrm{L} 12$ & run1 e run2 \\
\hline NASA-GISS-EH & Nasa Goddard Institute for Space Studies (NASA/GISS); Estados Unidos & $0.1 \mathrm{hPa} / 4^{\circ} \times 5^{\circ} \mathrm{L} 20$ & run1 a run5 \\
\hline NASA-GISS-ER & Nasa Goddard Institute for Space Studies (NASA/GISS); Estados Unidos & $0.1 \mathrm{hPa} / 4^{\circ} \times 5^{\circ} \mathrm{L} 20$ & run1 a run9 \\
\hline NCAR-CCSM3 & National Center for Atmospheric Research (NCAR); Estados Unidos & $2.2 \mathrm{hPa} / \mathrm{T} 85\left(1.4^{\circ} \times 1.4^{\circ}\right) \mathrm{L} 26$ & run1 a run7 e run9 \\
\hline NCAR-PCM & National Center for Atmospheric Research (NCAR), NSF, DOE, NASA, e NOAA; Estados Unidos & $2.2 \mathrm{hPa} / \mathrm{T} 42\left(\sim 2.8^{\circ} \times 2.8^{\circ}\right) \mathrm{L} 26$ & run1 a run4 \\
\hline NIES-MIROC3.2-HI & CCSR/ NIES/ FRCGC; Japão & $40 \mathrm{~km} \mathrm{~T} 106\left(\sim 1.1^{\circ} \times 1.1^{\circ}\right) \mathrm{L} 56$ & run1 \\
\hline NIES-MIROC3.2-MED & CCSR/ NIES/ FRCGC; Japão & $30 \mathrm{~km} \mathrm{~T} 42\left(\sim 2.8^{\circ} \times 2.8^{\circ}\right) \mathrm{L} 20$ & run1 a run3 \\
\hline UKMO-HADCM3 & Hadley Centre for Climatic Prediction and Research, Met Office; ReinoUnido & $5 \mathrm{hPa} / 2.5^{\circ} \times 3.75^{\circ} \mathrm{L} 19$ & run1 e run2 \\
\hline UKMO-HADGEM1 & Hadley Centre for Climatic Prediction and Research, Met Office; ReinoUnido & $39.2 \mathrm{~km} / \sim 1.3^{\circ} \times 1.9^{\circ} \mathrm{L} 38$ & run1 \\
\hline
\end{tabular}




\section{Dados observacionais}

A base de dados observacionais utilizada para verificar a destreza dos modelos do IPCC sobre o continente é proveniente da University of East Anglia/Climate Research Unit (CRU) (NEW; HULME; JONES, 1999; NEW et al., 2001) (http://badc.nerc. ac.uk/data/cru/), enquanto sobre o oceano são usadas as reanálises 20th CenturyReanalysis V2 (COMPO; WHITAKER; SARDESHMUKH, 2006; WHITAKER et al., 2004), fornecidas pelo National Oceanic and Atmospheric Administration (NOAA) a partir do site http://www.esrl.noaa.gov/psd/.

O conjunto de dados utilizados consiste em séries temporais de precipitação obtidas por meio dos registros mensais medidos em milímetros ( $\mathrm{mm})$, desde janeiro de 1901 a dezembro de 1999, com resolução de 0,5 graus para o continente (CRU TS 3.0) e 2,0 graus para o oceano (20th Century Reanalysis V2).

\section{Modelos do IPCG}

Os dados provenientes do CMIP3 utilizados pelo IPCC-AR4 são resultados de simulações de modelos globais de alguns centros de pesquisa que contribuíram para esse relatório (conforme Tabela 1), forçadas pelas concentrações observadas de gases de efeito estufa durante o século XX (simulações do cenário 20C3M).

Para avaliação dos modelos do IPCC-AR4 foram consideradas todas as simulações disponíveis de cada um dos modelos, totalizando 73 simulações.

Neste trabalho o nome de cada uma das simulações dos modelos do IPCC-AR4 será formado pela designação do modelo mais a rodada disponível. Por exemplo, para o modelo BCCR-BCM2 e rodada 1, tem-se : bccr.bcm2.run1.

\section{Critérios de Avaliação}

Em busca de identificar os modelos que melhor representam os padrões de variação do século XX foi adotada a avaliação sazonal proposta por Silveira et al. (2012) e uma adaptação da avaliação plurianual proposta por Lázaro (2011).

A avaliação é constituída por três etapas que analisam padrões de variação de diferentes escalas: sazonal, interanual e interdecadal.

Para avaliação sazonal $\left(\mathrm{AVAL}_{\mathrm{s}}\right)$ dos modelos foi calculada a climatologia mensal média sobre a região de estudo para todos os modelos do IPCC e a observação (CRU continente e NOAA oceano). Em seguida é feita uma comparação com base em al- guns índices estatísticos para que possam ser definidos que modelos possuem melhor comportamento para América do Sul.

As avaliações interanual ( $\left.\mathrm{AVAL}_{\mathrm{a}}\right)$ e interdecadal $\left(\mathrm{AVAL}_{\mathrm{d}}\right)$ baseiam-se na transformada de ondeletas (TORRENCE; COMPO, 1998), sobretudo na comparação do espectro global de variação das séries observadas e modeladas.

Cada uma das avaliações possui valores entre zero e 1, que representam, respectivamente, o pior modelo para todos os índices daquela análise e o melhor. Depois de calculado $\mathrm{AVAL}_{\mathrm{s}}, \mathrm{AVAL}_{\mathrm{a}}$ e A$\mathrm{VAL}_{\mathrm{d}}$ de todos os modelos é sugerido um índice geral, $\mathrm{AVAL}_{\mathrm{t}}$, conforme equações 1 e 2. Este é dado pelo somatório das avaliações, ponderados por um valor $\gamma_{\mathrm{j}}$ (que possui valores entre zero e 1 ).

A ponderação pode ser feita de acordo com os interesses do avaliador: ao atribuir valores iguais para $\gamma_{j}$ todas as avaliações possuem o mesmo peso no índice geral, enquanto ao atribuir valores diferentes as três avaliações possuirão pesos distintos na análise geral.

A avaliação geral dos modelos do IPCC-AR4 segue o modelo hierárquico das equações 1 e 2:

$$
\begin{aligned}
& A V A L_{y}=\sum_{j=1} \gamma_{j} \cdot A V A L_{j} \\
& \sum_{j=1}^{j=N} \gamma_{j}=1
\end{aligned}
$$

Na figura 3 são mostrados os critérios de avaliação dos modelos do IPCC-AR4 para o período de 1901 a 1999.

Critérios de Avaliação do Desempenho dos modelos do IPCC quanto à representação da Climatologia Média (Avaliação Sazonal)

A representação da sazonalidade é de grande relevância para a avaliação dos impactos do clima em recursos hídricos e agricultura. O início do plantio das culturas é o regime fluvial são condicionados pela distribuição temporal das chuvas. Uma má representação desta sazonalidade compromete a avaliação dos impactos das mudanças climáticas sobre estas duas importantes áreas. Adicionalmente podese avaliar que os totais de precipitações mensais e sua sazonalidade são considerados como indicativo da qualidade que o modelo tem de representar os sistemas geradores de chuvas e sua ocorrência. 


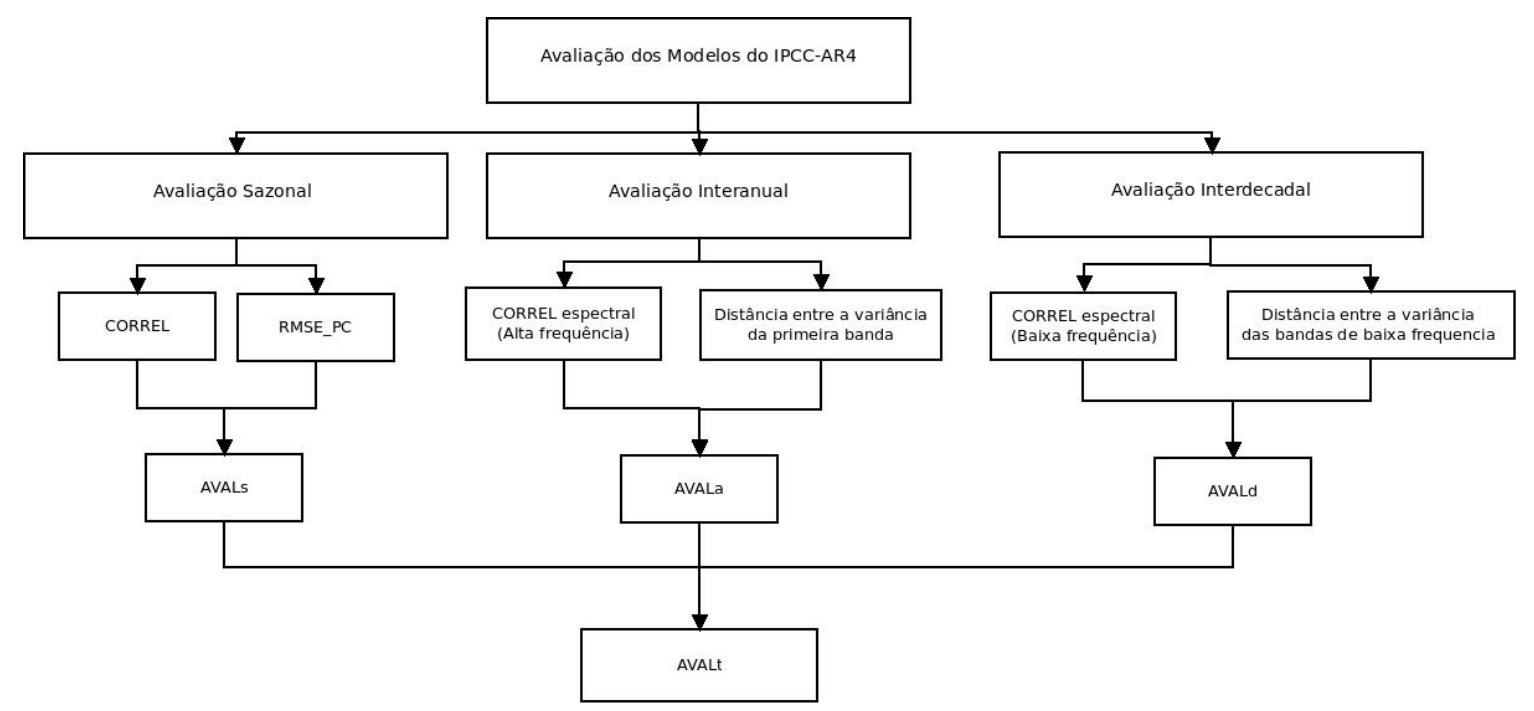

Figura 3 - Critérios de avaliação dos modelos do IPCC-AR4 no período de 1901 a 1999

A avaliação sazonal $\left(\mathrm{AVAL}_{\mathrm{s}}\right)$ dos modelos é baseada na comparação entre a climatologia mensal média sobre a região de estudo para todos os modelos do IPCC e a observação (CRU continente e NOAA oceano).

As medidas estatísticas utilizadas, cujas definições estão indicadas a seguir, são: raiz do erro quadrático médio da contribuição percentual mensal em relação às chuvas anuais (RMSE_PC) e correlação (CORREL) (WILKS, 1995).

A raiz do erro quadrático médio percentual (RMSE_PC) é a raiz quadrada da média das diferenças individuais quadráticas entre a contribuição percentual mensal das chuvas modeladas nos totais anuais e a contribuição percentual mensal das chuvas observadas nos totais anuais e é definida pela equação:

$R M S E_{-} P C=\sqrt{\frac{1}{n} \sum_{i=1}^{n}\left(\frac{100 . P_{i}}{\sum_{j=1}^{n} P_{j}}-\frac{100 . A_{i}}{\sum_{j=1}^{n} A_{j}}\right)^{2}}$,

onde $n$ são os meses, $P$ é projeção de cada mês e $A$ a análise considerada como observação.

Valores grandes do RMSE_PC representam grandes erros nos campos projetados, e valores próximos de zero indicam uma projeções quase perfeita. Elevando ao quadrado o termo da diferença, o RMSE_PG tende a dar maior peso às grandes discrepâncias entre os campos observados e previstos.
A correlação (equação 4) pode assumir valores entre - 1 e 1 que indicam, respectivamente, perfeita anticorrelação e perfeita correlação, havendo ainda a total ausência de correlação verificada com um resultado igual a zero. Este índice tem a capacidade de detectar correspondência de fase entre as séries, sendo, por construção, insensível a erros de viés.

$$
\text { CORREL }=\frac{\sum_{i=1}^{i=n}\left(A_{i}-\bar{A}\right)\left(P_{i}-\bar{P}\right)}{\sqrt{\sum_{i=1}^{i=n}\left(P_{i}-\bar{P}\right)^{2} \sum_{i=1}^{i=n}\left(A_{i}-\bar{A}\right)^{2}}} .
$$

Ao final do cálculo desses dois índices é feita uma avaliação ponderada para que os modelos possam ser classificados, dada por:

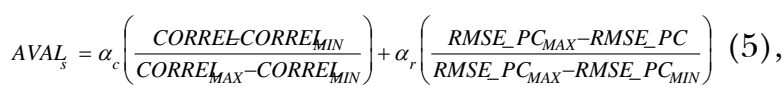

tal que

$\alpha_{c}+\alpha_{r}=1$

Sendo, CORREL $L_{\mathrm{MIN}}$ a menor correlação obtida entre os modelos do IPCC e CORREL ${ }_{\text {MAX }}$ a má-

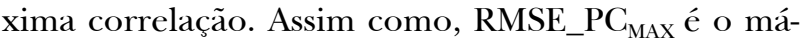
ximo erro quadrático médio percentual dos modelos do IPCC e RMSE_PC ${ }_{\text {MIN }}$ o mínimo. 
As variáveis $\alpha_{\mathrm{c}}$ e $\alpha_{\mathrm{r}}$ assumem valores entre 0 e 1 (conforme equação 6). Para $\alpha_{c}>\alpha_{r}$, a correlação exerce maior influência na avaliação do modelo, enquanto que para $\alpha_{\mathrm{r}}>\alpha_{\mathrm{c}}$ o RMSE_PC dos modelos possui maior peso na avaliação. Já para $\alpha_{\mathrm{r}}=\alpha_{\mathrm{c}}=0,5$, as duas métricas utilizadas neste trabalho exercem o mesmo efeito sobre o valor de AVAL.

A variável $\mathrm{AVAL}_{\mathrm{s}}$ assume valores entre 0 e 1 que indicam, respectivamente, o pior entre os modelos avaliados e o melhor deles, segundo este critério.

Critérios de Avaliação do Desempenho dos modelos do IPCC quanto à representação da variabilidade interanual e interdecadal.

Segundo Wilks (1995), entre outros, existem duas formas fundamentais de análise de series temporais quanto à variabilidade interanual e interdecadal: análises no domínio do tempo e no domínio de frequência. A primeira busca caracterizar a série de dados considerando o mesmo domínio em que os dados foram coletados/observados. Já as análises no domínio de frequência buscam a representação da série temporal em termos da contribuição de cada escala temporal para um dado valor.

Diante da dificuldade em se caracterizar as variabilidades tanto de espaço como de tempo no padrão de chuvas, torna-se necessário utilizar um método de análise adequado na localização temporal das estruturas de multiescalas. O método usado neste estudo para caracterizar as variabilidades tanto no espaço como no tempo é a Transformada em Ondeletas (TO). Este método é recomendado para o estudo de fenômenos transientes, espacialmente heterogêneos e que atuam simultaneamente em várias escalas do escoamento (TORRENCE; COMPO, 1998).

\section{Tranformada em ondoletas}

As ondeletas são funções matemáticas que caracterizam os padrões de variação de uma série de dados, em diferentes componentes de frequência, permitindo a análise de cada componente em sua escala correspondente. A análise da ondeleta mantém a localização do tempo e da frequência, em uma análise de sinal, pela decomposição ou transformação de uma série temporal unidimensional numa série difusa de tempo e frequência, simultaneamente. Assim, é possível obter informações da amplitude de quaisquer sinais periódicos dentro da série, bem como informações de como esta amplitude varia com o tempo.
Segundo Torrence e Compo (1998), a transformada em ondeletas tem se tornado um método bastante comum na análise de picos localizados de variância ou potência em uma série temporal. Por meio da decomposição desta série temporal em diferentes espaços de tempo e frequência é possível determinar picos de potência em diferentes frequências e períodos. Esta transformada tem sido recomendada para alguns eventos em detrimento da transformada de Fourier (ROCHA, 2008).

Ao utilizar série de Fourier para transformar um dado sinal do domínio do tempo para o domínio da frequência, perde-se totalmente a informação sobre a localização temporal. Além disso, a partir da transformada de Fourier de um sinal, é impossível dizer onde um evento em particular está localizado, pois o que é obtido são as frequências que compõem o sinal. Isto faz com que a aplicação da Análise de Fourier se restrinja às séries com comportamento estacionário, ou ainda em problemas lineares ou funções periódicas.

A transformada contínua de ondeleta (TCO) é definida em termos de uma integral do produto da série analisada $f(t)$ e uma ondeleta conhecida como "ondeleta-mãe" $\psi_{a, b}(t)$, expressa pela equação 7:

$\operatorname{TCO}(\mathbf{a}, \mathbf{b})=\psi(t)=\int_{-\infty}^{+\infty} f(t) \psi_{a, b}(t) d t$

em que os parâmetros a e b variam continuamente em $\mathrm{R}$, com $\mathrm{a} \neq 0$, e

$\psi_{a, b}(t)=\frac{1}{\sqrt{a}} \psi\left(\frac{t-b}{a}\right) \quad \mathrm{a} \in \mathrm{R}+\mathrm{e} \mathrm{b} \in \mathrm{R}$

Sendo as funções ondoletas filhas geradas a partir de dilatações e translações da ondoleta-mãe $\psi_{\mathrm{a}, \mathrm{b}}(\mathrm{t})$. Na equação 8 os parâmetros a e b, correspondem, respectivamente, as informações de escala e translação.

A função ondoleta considerada para a análise foi a de Morlet, dada pela equação 9. Esta pertence à família de ondoletas complexas não-ortogonais e é definida pela equação 9:

$\psi(\eta)=\pi^{-1 / 4} e^{i w} 0^{\eta} e^{-\eta^{2} / 2}$

sendo $w_{0}=6$ e $\eta=t / s$ 
onde $t$ é o tempo e $s$ é a escala da ondeleta e $w_{0}$ é uma frequência não dimensional, que representa uma onda modulada por um envelope Gaussia$\operatorname{no}\left(e^{-\eta^{2} / 2}\right)$. Maiores detalhes podem ser encontrados em Boggess e Narcowich (2009), Ruch e Fleet (2009) e Torrence e Compo (1998).

Para identificar as frequências de variação mais significativas de uma série temporal pode-se utilizar a potência do sinal do Especto Global da Ondeleta. A potência está associada da intensidade do sinal da série história para uma dada frequência ou banda (intervalo) de frequências. A potência é o valor absoluto (norma da parte real e imaginária da ondeleta) ao quadrado dos valores da transformada da ondeleta da série temporal para cada frequência em determinado instante de tempo (TORRENCE; COMPO, 1998). Este procedimento, conhecido na literatura científica pelo termo em inglês de Global Wavelet Spectrum (Espectro de Ondeleta Global), é uma forma similar do espectro de energia obtido via Transformada Rápida de Fourier.

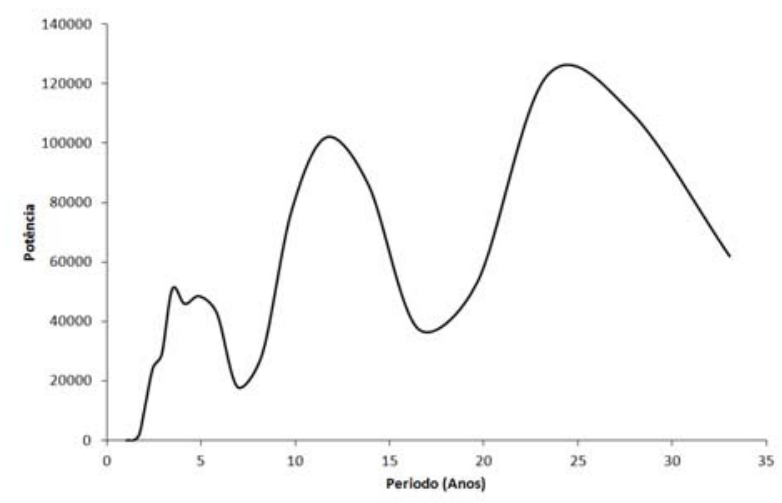

Figura 4- Espectro global de potência da região Nordeste Setentrional do Brasil (nNEB)

No espectro de energia global é possível identificar exatamente as bandas (frequências) características presentes na série analisada. Esta representação possui um caráter muito vantajoso e utilitário que é a localização no tempo da variabilidade da energia de uma série temporal. Nas figuras 4,5 e 6 , o eixo das abscissas refere-se ao período em anos, e o eixo das ordenadas representa a variância (energia) associada com cada período em anos. Maiores informações a respeito podem ser encontrados em Boggess e Narcowich (2009), Ruch e Fleet (2009) e Torrence e Compo (1998).

A transformada contínua de ondeletas envolve um enorme número de escalas o que gera um volume grande de dados. Uma estratégia computa- cionalmente mais eficiente é a escolha de apenas um subconjunto de escalas e posições para os quais os coeficientes devem ser calculados. Adotando-se essa estratégia, a transformada de ondeletas deixa de ser contínua para ser discreta. Este análise permite que um sinal seja representado como uma soma das componentes.

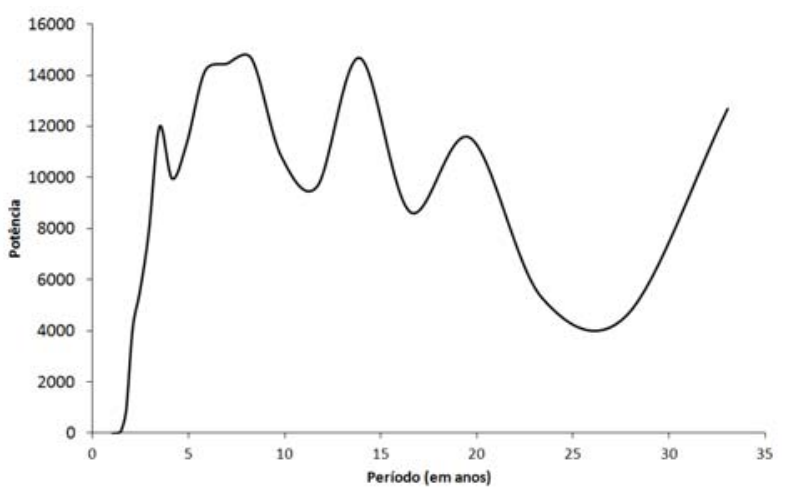

Figura 5 - Espectro global de potência da Bacia da Prata

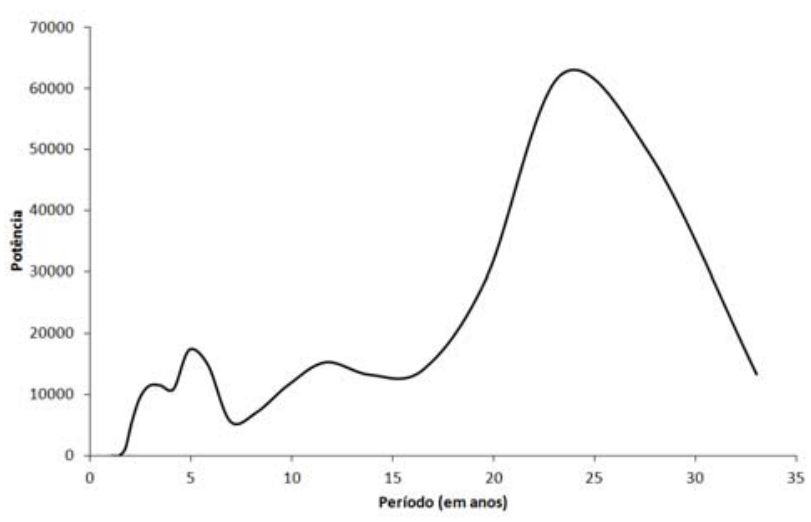

Figura 6 - Espectro global de potência da Amazônia

$f(t)=\sum_{i=1}^{n} W_{i}$

$i$ é o número de componentes e $\mathrm{W}_{\mathrm{i}}$ a ondeleta que representa a componente i.

\section{Critérios de Avaliação}

Utilizou-se como critério de avaliação plurianual a representação da série de dados observacionais de 1901 a 1999 da precipitação, através do espectro de energia global dos totais de precipitações anuais. A avaliação plurianual é dividida em duas: avaliação interanual e avaliação interdecadal. 
Para avaliação dos modelos é calculada o espectro de energia global sobre as regiões de estudo para todas as rodadas dos modelos do IPCC e as observações. Em seguida são identificados os principais padrões de variação das séries observadas a partir do Espectro Global da Ondeleta e a partir disto são executadas as seguintes etapas:

- decomposição do sinal para obter os coeficientes wavelets no domínio transformado. Para o caso da série observada de precipitação nas três regiões avaliadas a decomposição será igual à soma das bandas características no século XX e o resíduo. A partir da equação 11, tem-se:

$P(t)=R+\sum_{i=1}^{n} B_{i}$

$P(t)$ é a série de precipitação média anual observada;

$R$ é o resíduo;

$i$ é o número de bandas utilizadas;

$\mathrm{B}_{i}$ é a ondeleta que representa a banda $i$.

Cada uma das regiões avaliadas neste trabalho apresentou três bandas (conforme figuras 3, 4 e 5). Sendo assim, a primeira banda do espectro observado será considerada variação interanual e as demais regiões do espectro serão consideradas variação interdecadal.

- análise e processamento dos coeficientes neste domínio;

- reconstrução do sinal a partir dos coeficientes modificados.

O processo de reconstrução é repetido para os modelos do IPCC-AR4 para as mesmas bandas observadas. Em seguida, é feita uma comparação com base em alguns índices estatísticos para que possam ser definidos que modelos possuem melhor desempenho para a região de estudo.

As medidas estatísticas utilizadas, cujas definições estão indicadas a seguir, são: correlação entre a reconstituição da série do modelo e da observação para uma dada banda de frequência (CORREL) e a distância da variabilidade das variâncias das rodadas dos modelos (DIST).

Sendo as frações entre a variância espectral da banda e a variância do espectro global da série da chuva anual $f_{P}$ e $f_{A}$, tais que

$$
\begin{gathered}
f_{p}=\frac{V P_{s}}{V P_{G}} \\
f_{a}=\frac{V A_{s}}{V A_{G}}
\end{gathered}
$$

onde $\mathrm{VP}_{\mathrm{s}}$ é a variância da projeção de precipitação

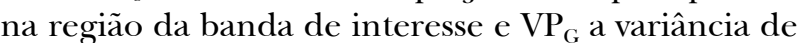
todo o espectro global de potência da série modelada. Onde $V A_{s}$ é a variância da análise na região significante e a $\mathrm{VA}_{\mathrm{G}}$ a variância do espectro global de ondeletas da observação.

A distância euclidiana DIST indica a variabilidade das variâncias das rodadas dos modelos quanto às variâncias dos dados observados por bandas e é definida pela equação:

$$
\operatorname{DIST}^{2}=\sum_{i=1}^{n}\left(f_{p_{i}}-f_{a_{i}}\right)^{2}
$$

$i$ é o número de bandas avaliado.

Valores grandes de DIST indicam uma distância maior entre as variâncias das modelos quanto à variância da série observada. Isto implica que o modelo possui dificuldade de representar a variabilidade da banda.

Para avaliação interanual é usada a correlação entre o espectro observado e modelado no período que vai do início do espectro ao fim da primeira banda associado ao índice DIST da primeira banda. Enquanto para avaliação interdecadal é usada a correlação entre o espectro observado e modelado no período que vai desde o fim da primeira banda ao final do espectro associado ao índice DIST da duas últimas bandas.

Ao final do cálculo desses índices é feita uma avaliação ponderada para representação interanual e outra para interdecadal, conforme equações $14,15,16$ e 17 , para que os modelos possam ser classificados. A avaliação interanual é dada por:

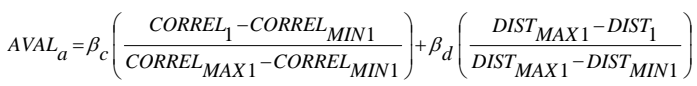

tal que

$\beta_{C}+\beta_{d}=1$

Sendo, CORREL $L_{\text {MIN1 }}$ a menor correlação obtida entre os modelos do IPCC e CORREL $L_{\text {MAX } 1}$ a 
Avaliação de Desempenho dos Modelos de Mudança Climática do IPCC-AR4 Quanto a Sazonalidade e os Padrões de Variabilidade Interanual da Precipitação Sobre o Nordeste do Brasil, Bacia do Prata e Amazônia

Tabela 2- Melhores e piores valores obtidos pelos índices estatísticos propostos para avaliação dos modelos do IPCC-AR4 na escala sazonal, interanual e interdecadal no Nordeste Setentrional do Brasil

\begin{tabular}{|c|c|c|c|c|c|}
\hline & Índices & Melhor & Modelo & Pior & Modelo \\
\hline Sazonal & $\begin{array}{l}\text { CORREL } \\
\text { RMSE_PG }\end{array}$ & $\begin{array}{l}0,981 \\
1,378 \\
\end{array}$ & $\begin{array}{c}\text { ipsl_cm4_run1 } \\
\text { mri_cgcm2_3_2a_run3 }\end{array}$ & $\begin{array}{l}0,142 \\
6,960 \\
\end{array}$ & $\begin{array}{l}\text { ncar_pcm1_run2 } \\
\text { ncar_pcm1_run1 }\end{array}$ \\
\hline Interannual & $\begin{array}{c}\text { CORREL } \\
\text { DIST }\end{array}$ & $\begin{array}{l}0,984 \\
0,004 \\
\end{array}$ & $\begin{array}{c}\text { cnrm_cm3_run1 } \\
\text { cccma_cgcm3_1_run3 }\end{array}$ & $\begin{array}{l}0,418 \\
0,834 \\
\end{array}$ & $\begin{array}{c}\text { giss_model_e_h_run5 } \\
\text { csiro_mk3_5_run1 }\end{array}$ \\
\hline Interdecadal & $\begin{array}{c}\text { CORREL } \\
\text { DIST }\end{array}$ & $\begin{array}{l}0,864 \\
0,163\end{array}$ & $\begin{array}{c}\text { giss_model_e_r_run8 } \\
\text { csiro_mk3_0_run2 }\end{array}$ & $\begin{array}{r}-0,734 \\
1,226\end{array}$ & $\begin{array}{l}\text { mpi_echam5_run3 } \\
\text { mpi_echam5_run3 }\end{array}$ \\
\hline
\end{tabular}

máxima correlação em relação ao observado na primeira região do espectro avaliada. Assim como,

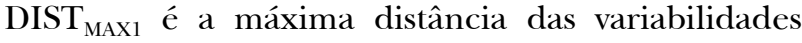
por bandas das rodadas dos modelos IPCC e DISTMIN O mínimo em relação à primeira região do espectro avaliada.

A avaliação interdecadal é dada por:

AVAL $_{d}=\sigma_{c}\left(\frac{\text { CORREL }_{2}-\text { CORREL }_{M I N 2}}{\text { CORREL }_{M A X 2} 2^{-C O R R E L} L_{M I N 2}}\right)+\sigma_{d}\left(\frac{\text { DIST }_{M A X 2}-\text { DIST }_{2}}{\text { DIST }_{M A X 2} \text { DIST }_{\text {MIN } 2}}\right)$

$\sigma_{C}+\sigma_{d}=1$

Sendo, CORREL $\mathrm{MIN}_{\mathrm{M} 1}$ a menor correlação obtida entre os modelos do IPCC e CORREL $\mathrm{CAX}_{\mathrm{MA}}$ a máxima correlação em relação ao observado na primeira região do espectro avaliada. Assim como,

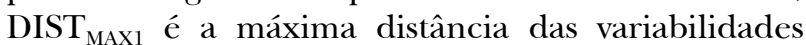
por bandas das rodadas dos modelos IPCC e DISTMIN O mínimo em relação a primeira região do espectro avaliada.

As variáveis $\beta_{\mathrm{c}}, \beta_{\mathrm{r}}, \sigma_{\mathrm{c}}$ e $\sigma_{\mathrm{r}}$ assumem valores entre 0 e 1 (conforme equações 15 e 17). Para valores $\beta_{\mathrm{c}}>\beta_{\mathrm{d}}$ e $\sigma_{\mathrm{c}}>\sigma_{\mathrm{r}}$, a correlação exerce maior influência na avaliação do modelo, enquanto que para $\beta_{\mathrm{c}}<\beta_{\mathrm{d}} \mathrm{e}$ $\sigma_{\mathrm{c}}<\sigma_{\mathrm{r}} \mathrm{o}$ índice DIST exerce maior efeito.

As variáveis $\mathrm{AVAL}_{\mathrm{y}}$ e $\mathrm{AVAL}_{\mathrm{d}}$ assumem valores entre 0 e 1 que indicam, respectivamente, o pior entre os modelos avaliados e o melhor deles, segundo este critério.

\section{RESULTADOS E DISCUSSÕES}

\section{Avaliação do Nordeste Setentrional do Brasil}

Na figura 4 é mostrado graficamente o espectro global da série observada na região Nordeste
Setentrional do Brasil. Notam-se nitidamente nessa região três padrões de variação: 3 a 6 anos, 9 a 14 anos e 19 a 33 anos.

Na tabela 2 são mostrados os melhores e piores valores obtidos pelos índices estatísticos propostos para avaliação dos modelos do IPCC-AR4 na escala sazonal, interanual e interdecadal para o Nordeste Setentrional do Brasil.

Os modelos indicam uma boa representação dos padrões de variação interanual, com as correlações espectrais da primeira banda entre 0,984 e 0,418. Entretanto a maioria dos modelos possui muita dificuldade de representar as variações interdecadais, conforme indica a correlação espectral negativa de $-0,734$ para segunda e terceira banda de variação do pior dos modelos e o AVALd da tabela 3 que indica que apenas 8 modelos obtiveram valores maiores que 0,7 .

O modelo NCAR.PCM1.RUN2 apresentou a pior correlação sazonal, enquanto o modelo NCAR.PCM1.RUN1 o pior RMSE. Considerando que estes modelos apresentaram AVALs aproximadamente zero e que os demais indicaram valores superiores a 0,5 (exceto NCAR.PCM1.RUN3), as rodadas 1,2 e 3 do modelo NCAR.PCM1 é inferior aos demais na representação da variações sazonais da precipitação.

Na tabela 3 são mostrados os valores obtidos para avaliação sazonal, interanual, interdecadal e total de todos os modelos do IPCC-AR4 em ordem de classificação, para $\gamma_{c}=\gamma_{r}=\gamma_{e}=1 / 3$ para o Nordeste Setentrional do Brasil. O modelo CSIRO.MK3.0.run2 obteve o maior AVALt, seguido pelos modelos GISS.MODEL.E.R.RUN8 e UKMO.HADCM3.RUN1.

A maioria dos modelos representa adequadamente as variações sazonais e interanuais da precipitação, com mais da metade dos modelos indicando AVALs e AVALa superiores a 0,75. 
Tabela 3- Valores obtidos para avaliação sazonal, interanual, interdecadal e total de todos os modelos do IPCC-AR4 em ordem de classificação, para $\gamma_{\mathrm{c}}=\gamma_{\mathrm{r}}=\gamma_{\mathrm{e}}=1 / 3$ para o Nordeste Setentrional do Brasil

\begin{tabular}{|c|c|c|c|c|c|c|c|c|c|c|c|}
\hline Global Models & CLAS & AVALs & AVALa & AVALd & AVALt & Global Models & CLAS & AVALs & AVALa & AVALd & AVALt \\
\hline csiro.mk3.0.run2 & 1 & 0,785 & 0,874 & 0,950 & 0,870 & csiro.mk3.5.run3 & 38 & 0,690 & 0,835 & 0,328 & 0,618 \\
\hline giss.model.e.r.run8 & 2 & 0,909 & 0,779 & 0,849 & 0,846 & csiro.mk3.0.run3 & 39 & 0,762 & 0,606 & 0,474 & 0,614 \\
\hline ukmo.hadcm3.run1 & 3 & 0,805 & 0,914 & 0,798 & 0,839 & gfdl.cm2.1.run1 & 40 & 0,834 & 0,771 & 0,216 & 0,607 \\
\hline mri.cgcm2.3.2a.run1 & 4 & 0,991 & 0,799 & 0,593 & 0,794 & giss.model.e.r.run9 & 41 & 0,904 & 0,451 & 0,466 & 0,607 \\
\hline csiro.mk3.0.run1 & 5 & 0,790 & 0,798 & 0,788 & 0,792 & gfdl.cm2.0.run1 & 42 & 0,766 & 0,803 & 0,242 & 0,604 \\
\hline giss.model.e.r.run2 & 6 & 0,901 & 0,766 & 0,701 & 0,789 & ipsl.cm4.run1 & 43 & 0,901 & 0,634 & 0,269 & 0,601 \\
\hline mri.cgcm2.3.2a.run3 & 7 & 0,994 & 0,673 & 0,649 & 0,772 & mri.cgcm2.3.2a.run4 & 44 & 0,691 & 0,797 & 0,311 & 0,600 \\
\hline cnrm.cm3.run1 & 8 & 0,925 & 0,816 & 0,546 & 0,762 & ncar.ccsm3.0.run7 & 45 & 0,651 & 0,807 & 0,339 & 0,599 \\
\hline giss.model.e.r.run6 & 9 & 0,891 & 0,710 & 0,686 & 0,762 & iap.fgoals1.0.g.run2 & 46 & 0,875 & 0,547 & 0,369 & 0,597 \\
\hline cccma.cgcm3.1.run3 & 10 & 0,793 & 0,884 & 0,575 & 0,751 & cccma.cgcm3.1.run5 & 47 & 0,799 & 0,687 & 0,306 & 0,597 \\
\hline miroc3.2.medres.run1 & 11 & 0,764 & 0,593 & 0,891 & 0,750 & gfdl.cm2.0.run3 & 48 & 0,772 & 0,835 & 0,180 & 0,596 \\
\hline giss.model.e.r.run5 & 12 & 0,913 & 0,798 & 0,530 & 0,747 & cccma.cgcm3.1.run4 & 49 & 0,776 & 0,602 & 0,408 & 0,595 \\
\hline miroc3.2.medres.run2 & 13 & 0,748 & 0,913 & 0,545 & 0,735 & iap.fgoals1.0.g.run3 & 50 & 0,869 & 0,549 & 0,359 & 0,592 \\
\hline giss.model.e.r.run3 & 14 & 0,904 & 0,903 & 0,381 & 0,729 & miub.echo.g.run4 & 51 & 0,546 & 0,764 & 0,458 & 0,589 \\
\hline cccma.cgcm3.1.run 1 & 15 & 0,784 & 0,808 & 0,573 & 0,722 & ncar.ccsm3.0.run2 & 52 & 0,675 & 0,893 & 0,197 & 0,588 \\
\hline giss.model.e.h.run3 & 16 & 0,883 & 0,848 & 0,430 & 0,721 & gfdl.cm2.0.run2 & 53 & 0,780 & 0,678 & 0,292 & 0,583 \\
\hline bccr.bcm2.0.run1 & 17 & 0,953 & 0,798 & 0,394 & 0,715 & inmcm3.0.run 1 & 54 & 0,706 & 0,691 & 0,348 & 0,581 \\
\hline mpi.echam5.run4 & 18 & 0,566 & 0,880 & 0,672 & 0,706 & ncar.ccsm3.0.run4 & 55 & 0,668 & 0,798 & 0,263 & 0,576 \\
\hline giss.model.e.r.run1 & 19 & 0,909 & 0,756 & 0,433 & 0,700 & ncar.ccsm3.0.run3 & 56 & 0,663 & 0,806 & 0,257 & 0,575 \\
\hline giss.aom.run1 & 20 & 0,586 & 0,755 & 0,746 & 0,695 & mpi.echam5.run2 & 57 & 0,567 & 0,818 & 0,341 & 0,575 \\
\hline ncar.ccsm3.0.run5 & 21 & 0,674 & 0,847 & 0,552 & 0,691 & mpi.echam5.run1 & 58 & 0,582 & 0,764 & 0,355 & 0,567 \\
\hline giss.model.e.h.run2 & 22 & 0,860 & 0,866 & 0,345 & 0,690 & ukmo.hadcm3.run2 & 59 & 0,869 & 0,348 & 0,436 & 0,551 \\
\hline giss.model.e.r.run4 & 23 & 0,903 & 0,805 & 0,361 & 0,690 & miroc3.2.hires.run1 & 60 & 0,792 & 0,450 & 0,380 & 0,541 \\
\hline miroc3.2.medres.run3 & 24 & 0,763 & 0,648 & 0,633 & 0,681 & miub.echo.g.run3 & 61 & 0,562 & 0,584 & 0,461 & 0,536 \\
\hline giss.model.e.h.run1 & 25 & 0,860 & 0,781 & 0,394 & 0,678 & ncar.ccsm3.0.run1 & 62 & 0,676 & 0,665 & 0,265 & 0,535 \\
\hline giss.model.e.r.run7 & 26 & 0,908 & 0,826 & 0,293 & 0,676 & ukmo.hadgem1.run1 & 63 & 0,684 & 0,494 & 0,423 & 0,534 \\
\hline mri.cgcm2.3.2a.run2 & 27 & 0,990 & 0,871 & 0,154 & 0,672 & ncar.ccsm3.0.run6 & 64 & 0,676 & 0,645 & 0,263 & 0,528 \\
\hline gfdl.cm2.1.run2 & 28 & 0,830 & 0,902 & 0,268 & 0,667 & ncar.pcm1.run2 & 65 & 0,001 & 0,673 & 0,805 & 0,493 \\
\hline cccma.cgcm3.1.t63.run1 & 29 & 0,797 & 0,540 & 0,657 & 0,665 & miub.echo.g.run5 & 66 & 0,582 & 0,587 & 0,279 & 0,483 \\
\hline giss.model.e.h.run4 & 30 & 0,873 & 0,787 & 0,314 & 0,658 & csiro.mk3.5.run1 & 67 & 0,650 & 0,392 & 0,380 & 0,474 \\
\hline csiro.mk3.5.run2 & 31 & 0,683 & 0,786 & 0,500 & 0,656 & iap.fgoals1.0.g.run1 & 68 & 0,877 & 0,344 & 0,170 & 0,464 \\
\hline ncar.pcm1.run4 & 32 & 0,803 & 0,786 & 0,369 & 0,653 & miub.echo.g.run2 & 69 & 0,588 & 0,690 & 0,099 & 0,459 \\
\hline ingv.echam4.run1 & 33 & 0,808 & 0,772 & 0,373 & 0,651 & ncar.pcm1.run1 & 70 & 0,006 & 0,606 & 0,597 & 0,403 \\
\hline miub.echo.g.run1 & 34 & 0,599 & 0,837 & 0,496 & 0,644 & mpi.echam5.run3 & 71 & 0,592 & 0,573 & 0,000 & 0,388 \\
\hline cccma.cgcm3.1.run2 & 35 & 0,803 & 0,670 & 0,449 & 0,641 & ncar.ccsm3.0.run9 & 72 & 0,029 & 0,732 & 0,350 & 0,370 \\
\hline giss.model.e.h.run5 & 36 & 0,885 & 0,310 & 0,691 & 0,629 & ncar.pcm1.run3 & 73 & 0,026 & 0,793 & 0,139 & 0,319 \\
\hline giss.aom.run2 & 37 & 0,584 & 0,853 & 0,436 & 0,624 & & & & & & \\
\hline
\end{tabular}


Avaliação de Desempenho dos Modelos de Mudança Climática do IPCC-AR4 Quanto a Sazonalidade e os Padrões de Variabilidade Interanual da Precipitação Sobre o Nordeste do Brasil, Bacia do Prata e Amazônia

Tabela 4- Melhores e piores valores obtidos pelos índices estatísticos propostos para avaliação dos modelos do IPCC-AR4 na escala sazonal, interanual e interdecadal para Bacia da prata

\begin{tabular}{l|lll|ll}
\hline \hline \multicolumn{2}{l}{ Índices } & Melhor & Modelo & Pior & Modelo \\
\hline \multirow{2}{*}{ Sazonal } & CORREL & 0,993 & bccr_bcm2_0_run1 & 0,928 & mpi_echam5_run3 \\
& RMSE_PC & 0,481 & ukmo_hadgem1_run1 & 3,957 & gfdl_cm2_1_run1 \\
\hline \multirow{2}{*}{ Interannual } & CORREL & 0,972 & mpi_echam5_run2 & 0,058 & miub_echo_g_run5 \\
& DIST & 0,002 & giss_model_e_r_run2 & 0,591 & mpi_echam5_run3 \\
\hline \multirow{2}{*}{ Interdecadal } & CORREL & 0,791 & gfdl_cm2_0_run2 & $-0,812$ & ncar_ccsm3_0_run5 \\
& DIST & 0,036 & ncar_ccsm3_0_run9 & 1,934 & miub_echo_g_run2 \\
\hline
\end{tabular}

As rodadas 1,2 e 3 do modelo MRI.CGM2.3.2 apresentaram correlações superiores a 0,95 e RMSE_PC inferior a $1 \%$, por isso são consideradas as melhores representações sazonais do século XX segundo a avaliação proposta neste traba lho, seguido pelos modelos bccr.bcm2.0.run1 e cnrm.cm3.run1. Para maiores informações sobre a avaliação sazonal do Nordeste Setentrional do Brasil consulte Silveira et al. (2012).

Enquanto as rodadas 1,2 e 3 do modelo NCAR_PCM1 apresentaram correlações sazonais inferior a 0,2, bem abaixo dos demais modelos, e o maior RMSE_PC. Isto levou as rodadas desse modelo a apresentar o menor AVALs, indicando que o mesmo não representa adequadamente a sazonalidade da precipitação no Nordeste Setentrional brasileiro.

As rodadas dos modelos GISS_AOM, MIUB_ECHO_G e MPI_ECHAM5 apresentaram correlações sazonais inferiores aos demais, associados a um RMSE_PC superior a 3,5\%, por isso obtiveram um AVALs bastante baixo.

Quanto a avaliação interanual o modelo UKMO_HADCM3_RUN1 obteve o melhor AVALa, enquanto o modelo CSIRO_M_K_3_5_RUN2 obteve o melhor AVALd. A maioria dos modelos do IPCC apresentou correlação espectral interdecadal negativa, indicando que os mesmos possuem muita dificuldade de representar este padrão de variação, principalmente as rodadas dos modelos NCAR.CCSM3.0, GFDL.CM.2.0 e GFDL.CM.2.1.

\section{Avaliação da Bacia da Prata}

Na figura 5 é mostrado graficamente o espectro global da série observada na Bacia da Prata. Notam-se nitidamente nessa região três padrões de variação: 2,5 a 9,8 anos, 11,6 a 14 anos e 16,5 a 23,4 anos.
Na tabela 4 são mostrados os melhores e piores valores obtidos pelos índices estatísticos propostos para avaliação dos modelos do IPCC-AR4 na escala sazonal, interanual e interdecadal para Bacia da Prata.

Os modelos representam muito bem os padrões de variação sazonal com correlações que variam de 0,928 a 0,993 e RMSE_PC variando de 0,481 a 3,957. Considerando que 20 modelos obtiveram AVALd maior que 0,7 , a grande maioria possui muita dificuldade de representar as variações decadais.

Na tabela 5 são mostrados os valores obtidos para avaliação sazonal, interanual, interdecadal e total de todos os modelos do IPCC-AR4 em ordem de classificação, para $\gamma_{c}=\gamma_{r}=\gamma_{e}=1 / 3$ para a Bacia da Prata. Entre os cinco primeiros modelos existem quatro rodadas do modelo cccma_cgcm3_1, indicando que este capta muito bem os padrões de variação da série para essa região no século XX.

As rodadas do modelo NCAR_PCM1 estão entre os piores modelos no NEB e na Bacia da Prata e Amazônia, considerando a avaliação sazonal proposta neste trabalho. Portanto esse modelo mostrase inadequado para representar a climatologia média para as regiões analisadas.

O modelo ukmo_hadgem1_run1 apresentou $\mathrm{AVAL}_{\mathrm{s}}$ igual a 0,942 , bem superior aos demais que apresentaram valores inferiores a 0,820. As Rodadas dos modelos CSIRO_MK3_5, GFDL_CM2_0, GFDL_CM2_1, NCAR_CCSM_1_0 e IPSL_CM4 apresentaram $\mathrm{AVAL}_{\mathrm{s}}$ inferiores aos demais, por isso obtiveram uma classificação bastante baixa.

Para bacia da Prata a maioria dos modelos possui uma boa representação da banda interanual, porém as rodadas do modelo IAPG_FGOALS_G mostram-se bastante inferiores aos demais modelos, com AVALa inferior a 0,4. 
Tabela 5- Valores obtidos para avaliação sazonal, interanual, interdecadal e total de todos os modelos do IPCG-AR4 em ordem de classificação, para para $\gamma_{c}=\gamma_{r}=\gamma_{e}=1 / 3$ para a Bacia da Prata

\begin{tabular}{|c|c|c|c|c|c|c|c|c|c|c|c|}
\hline Global Models & CLAS & AVALs & AVALa & AVALd & AVALt & Global Models & CLAS & AVALs & AVALa & AVALd & AVALt \\
\hline cccma_cgcm3_1_run2 & 1 & 0,675 & 0,779 & 0,877 & 0,777 & giss_model_e_h_run4 & 38 & 0,621 & 0,808 & 0,408 & 0,613 \\
\hline cccma_cgcm3_1_run4 & 2 & 0,708 & 0,782 & 0,799 & 0,763 & gfdl_cm2_0_run1 & 39 & 0,267 & 0,829 & 0,711 & 0,603 \\
\hline ukmo_hadcm3_run2 & 3 & 0,644 & 0,763 & 0,830 & 0,745 & miroc3_2_medres_run3 & 40 & 0,559 & 0,829 & 0,418 & 0,602 \\
\hline cccma_cgcm3_1_run1 & 4 & 0,699 & 0,686 & 0,838 & 0,741 & giss_model_e_r_run3 & 41 & 0,462 & 0,757 & 0,586 & 0,601 \\
\hline cccma_cgcm3_1_run3 & 5 & 0,686 & 0,761 & 0,739 & 0,729 & mri_cgcm2_3_2a_run1 & 42 & 0,495 & 0,648 & 0,635 & 0,593 \\
\hline ukmo_hadgem1_run1 & 6 & 0,942 & 0,779 & 0,464 & 0,728 & miroc3_2_medres_run1 & 43 & 0,543 & 0,686 & 0,536 & 0,588 \\
\hline mpi_echam5_run4 & 7 & 0,647 & 0,657 & 0,840 & 0,715 & ingv_echam4_run1 & 44 & 0,708 & 0,500 & 0,554 & 0,588 \\
\hline miub_echo_g_run1 & 8 & 0,579 & 0,848 & 0,702 & 0,710 & ncar_ccsm3_0_run1 & 45 & 0,357 & 0,857 & 0,547 & 0,587 \\
\hline giss_model_e_h_run3 & 9 & 0,593 & 0,678 & 0,851 & 0,707 & csiro_mk3_0_run1 & 46 & 0,502 & 0,691 & 0,561 & 0,585 \\
\hline giss_aom_run2 & 10 & 0,812 & 0,721 & 0,555 & 0,696 & ukmo_hadcm3_run1 & 47 & 0,661 & 0,392 & 0,693 & 0,582 \\
\hline ncar_ccsm3_0_run4 & 11 & 0,397 & 0,911 & 0,772 & 0,693 & csiro_mk3_5_run2 & 48 & 0,306 & 0,862 & 0,574 & 0,581 \\
\hline giss_aom_run1 & 12 & 0,827 & 0,777 & 0,476 & 0,693 & giss_model_e_r_run1 & 49 & 0,449 & 0,705 & 0,581 & 0,578 \\
\hline giss_model_e_h_run1 & 13 & 0,611 & 0,801 & 0,648 & 0,686 & giss_model_e_r_run5 & 50 & 0,465 & 0,696 & 0,548 & 0,570 \\
\hline giss_model_e_h_run5 & 14 & 0,628 & 0,708 & 0,716 & 0,684 & iap_fgoals1_0_g_run3 & 51 & 0,588 & 0,366 & 0,752 & 0,569 \\
\hline gfdl_cm2_0_run2 & 15 & 0,308 & 0,781 & 0,959 & 0,683 & csiro_mk3_5_run3 & 52 & 0,256 & 0,731 & 0,720 & 0,569 \\
\hline giss_model_e_h_run2 & 16 & 0,646 & 0,896 & 0,476 & 0,673 & giss_model_e_r_run9 & 53 & 0,504 & 0,680 & 0,514 & 0,566 \\
\hline ncar_ccsm3_0_run7 & 17 & 0,400 & 0,810 & 0,805 & 0,672 & cccma_cgcm3_1_run5 & 54 & 0,687 & 0,696 & 0,305 & 0,563 \\
\hline mri_cgcm2_3_2a_run3 & 18 & 0,510 & 0,796 & 0,706 & 0,671 & mri_cgcm2_3_2a_run4 & 55 & 0,536 & 0,729 & 0,413 & 0,559 \\
\hline ncar_pcm1_run3 & 19 & 0,546 & 0,799 & 0,665 & 0,670 & miub_echo_g_run2 & 56 & 0,608 & 0,774 & 0,286 & 0,556 \\
\hline mpi_echam5_run1 & 20 & 0,563 & 0,762 & 0,678 & 0,668 & mpi_echam5_run2 & 57 & 0,428 & 0,675 & 0,532 & 0,545 \\
\hline cccma_cgcm3_1_t63_run1 & 21 & 0,815 & 0,593 & 0,593 & 0,667 & gfdl_cm2_1_run1 & 58 & 0,114 & 0,728 & 0,774 & 0,539 \\
\hline inmcm3_0_run1 & 22 & 0,627 & 0,847 & 0,526 & 0,667 & ipsl_cm4_run1 & 59 & 0,229 & 0,698 & 0,685 & 0,538 \\
\hline miub_echo_g_run4 & 23 & 0,610 & 0,739 & 0,640 & 0,663 & miub_echo_g_run3 & 60 & 0,594 & 0,556 & 0,461 & 0,537 \\
\hline ncar_pcm1_run1 & 24 & 0,436 & 0,717 & 0,819 & 0,658 & iap_fgoals1_0_g_run1 & 61 & 0,596 & 0,370 & 0,636 & 0,534 \\
\hline cnrm_cm3_run1 & 25 & 0,660 & 0,809 & 0,503 & 0,657 & ncar_pcm1_run4 & 62 & 0,412 & 0,585 & 0,590 & 0,529 \\
\hline bccr_bcm2_0_run1 & 26 & 0,780 & 0,718 & 0,470 & 0,656 & ncar_pcm1_run2 & 63 & 0,470 & 0,702 & 0,414 & 0,529 \\
\hline giss_model_e_r_run6 & 27 & 0,535 & 0,765 & 0,641 & 0,647 & csiro_mk3_0_run3 & 64 & 0,506 & 0,737 & 0,343 & 0,529 \\
\hline mri_cgcm2_3_2a_run2 & 28 & 0,483 & 0,762 & 0,678 & 0,641 & csiro_mk3_0_run2 & 65 & 0,473 & 0,575 & 0,513 & 0,520 \\
\hline giss_model_e_r_run2 & 29 & 0,412 & 0,930 & 0,569 & 0,637 & ncar_ccsm3_0_run3 & 66 & 0,387 & 0,836 & 0,329 & 0,517 \\
\hline miroc3_2_medres_run2 & 30 & 0,523 & 0,864 & 0,518 & 0,635 & gfdl_cm2_1_run2 & 67 & 0,118 & 0,834 & 0,589 & 0,514 \\
\hline miroc3_2_hires_run1 & 31 & 0,720 & 0,628 & 0,542 & 0,630 & miub_echo_g_run5 & 68 & 0,620 & 0,091 & 0,787 & 0,499 \\
\hline giss_model_e_r_run4 & 32 & 0,474 & 0,732 & 0,676 & 0,627 & ncar_ccsm3_0_run5 & 69 & 0,387 & 0,765 & 0,311 & 0,488 \\
\hline ncar_ccsm3_0_run2 & 33 & 0,425 & 0,820 & 0,633 & 0,626 & gfdl_cm2_0_run3 & 70 & 0,289 & 0,782 & 0,378 & 0,483 \\
\hline ncar_ccsm3_0_run9 & 34 & 0,383 & 0,870 & 0,619 & 0,624 & csiro_mk3_5_run1 & 71 & 0,280 & 0,529 & 0,597 & 0,469 \\
\hline giss_model_e_r_run8 & 35 & 0,490 & 0,649 & 0,727 & 0,622 & mpi_echam5_run3 & 72 & 0,421 & 0,478 & 0,486 & 0,462 \\
\hline ncar_ccsm3_0_run6 & 36 & 0,367 & 0,849 & 0,642 & 0,619 & iap_fgoals1_0_g_run2 & 73 & 0,582 & 0,217 & 0,473 & 0,424 \\
\hline giss_model_e_r_run7 & 37 & 0,398 & 0,810 & 0,640 & 0,616 & & & & & & \\
\hline
\end{tabular}


Avaliação de Desempenho dos Modelos de Mudança Climática do IPCC-AR4 Quanto a Sazonalidade e os Padrões de Variabilidade Interanual da Precipitação Sobre o Nordeste do Brasil, Bacia do Prata e Amazônia

Tabela 6- Melhores e piores valores obtidos pelos índices estatísticos propostos para avaliação dos modelos do IPCC-AR4 na escala sazonal, interanual e interdecadal para Amazônia

\begin{tabular}{l|lll|ll}
\hline \hline & Índices & Melhor & Modelo & Pior & Modelo \\
\hline \multirow{2}{*}{ Sazonal } & CORREL & 0,981 & giss_model_e_r_run3 & 0,009 & ncar_pcm1_run3 \\
& RMSE_PC & 0,889 & giss_model_e_h_run5 & 7,924 & csiro_mk3_5_run3 \\
\hline \multirow{2}{*}{ Interannual } & CORREL & 0,977 & giss_model_e_r_run7 & 0,408 & giss_model_e_h_run5 \\
& DIST & 0,002 & bccr_bcm2_0_run1 & 0,609 & iap_fgoals1_0_g_run2 \\
\hline \multirow{2}{*}{ Interdecadal } & CORREL & 0,229 & ncar_pcm1_run3 & $-0,404$ & cccma_cgcm3_1_run4 \\
& DIST & 0,297 & ingv_echam4_run1 & 1,663 & miroc3_2_medres_run3 \\
\hline
\end{tabular}

\section{Avaliação da região da Amazônia}

Na figura 6 é mostrado graficamente o espectro global da série observada na Amazônia. Notam-se nitidamente nessa região três padrões de variação: 2 a 7 anos, 8,2 a 13,9 anos e 16,5 a 33 anos. Sendo a última banda a mais energética, indicando que está possui grande influência na variabilidade da série.

Na tabela 6 são mostrados os melhores e piores valores obtidos pelos índices estatísticos propostos para avaliação dos modelos do IPCC-AR4 na escala sazonal, interanual e interdecadal para Amazônia. Os modelos indicam uma boa representação interanual com a correlação espectral da primeira banda variando entre 0,408 e 0,977, porém as variações interdecadais não são tão bem representadas com correlações que variam de 0,229 a -0,404. Enquanto sazonalmente a maioria dos modelos possui boa representação com 67 modelos apontando correlações superiores a 0,60 .

Na tabela 7 são mostrados os valores obtidos para avaliação sazonal, interanual, interdecadal e total de todos os modelos do IPCC-AR4 em ordem de classificação, para $\gamma_{\mathrm{c}}=\gamma_{\mathrm{r}}=\gamma_{\mathrm{e}}=1 / 3$ para a Amazônia. Várias rodadas do modelo GISS_MODEL_E_R encontram-se entre os 20 primeiros colocados, com AVALt superior a 0,800 , isto indica que as rodadas desse modelo captam bem os padrões de variação da região. Enquanto as rodadas 1 e 2 do IAP_FGOALS_1_0 apresentaram o menor AVALt para a região, com bastante dificuldade de representar o padrão interanual de variação com AVALa inferior a 0,2 .

As rodadas dos modelos MIUB_ECHO_G e CSIRO_MK_3_5 apresentaram AVALs inferiores a 0,7 , mostrando que esses modelos são inferiores aos demais ao representar os padrões de variação sazonal. Além disso, as rodadas do modelo
NCAR_CCSM_3_0 apresentaram correlação inferior a 0,7 , bem inferior a maioria dos modelos.

\section{CONCLUSÕES}

A avaliação proposta neste trabalho identificou os melhores e piores modelos na representação dos padrões de variação da precipitação no século XX para o Nordeste Setentrional do Brasil, Amazônia e Bacia da Prata. Essas informações podem ser usadas pelos gestores em busca da escolha das projeções mais prováveis para o século XXI e pelos cientistas para um possível tratamento estatístico do conjunto das projeções. Este último grupo pode usar os resultados deste trabalho para avaliar o impacto nas vazões e evapotranspiração com maior número de modelos possíveis para tratar e dimensionar a incerteza existente nas projeções climáticas

Quanto à avaliação sazonal dos modelos IPCC algumas observações são destacadas:

- A maioria dos modelos globais do IPCC-AR4 apresentou correlações elevadas em relação à climatologia observada no período de 1901 a 1999 para as regiões avaliadas, mostrando que os modelos são capazes de capturar os padrões de variações sazonais.

- Os melhores modelos segundo a representação sazonal são: no Nordeste Setentrional do Brasil as rodadas do MRI.CGM2.3.2; na Bacia da PRATA o modelo UKMO_HADGEM1_RUN1 que apresentou $\mathrm{AVAL}_{\mathrm{s}}$ igual a 0,942; e na Amazônia as rodadas do GISS_MODEL_E_R;

- O modelo NCAR-PCM1 não representa adequadamente a climatologia média das três regiões;

- Na região do Nordeste Setentrional os modelos MPI-ECHAM5, MIUB-ECHO-G e 
Tabela 7- Valores obtidos para avaliação sazonal, interanual, interdecadal e total de todos os modelos do IPCC-AR4 em ordem de classificação, para $\gamma_{c}=\gamma_{r}=\gamma_{e}=1 / 3$ para a Amazônia

\begin{tabular}{|c|c|c|c|c|c|c|c|c|c|c|c|}
\hline Global Models & CLAS & AVALs & AVALa & AVALd & AVALt & Global Models & CLAS & AVALs & AVALa & AVALd & AVAL \\
\hline csiro_mk3_0_run1 & 1 & 0,954 & 0,781 & 0,956 & 0,897 & cccma_cgcm3_1_run1 & 38 & 0,845 & 0,825 & 0,591 & 0,753 \\
\hline ukmo_hadgem1_run1 & 2 & 0,956 & 0,923 & 0,784 & 0,888 & miub_echo_g_run3 & 39 & 0,699 & 0,744 & 0,816 & 0,753 \\
\hline giss_model_e_r_run1 & 3 & 0,882 & 0,874 & 0,897 & 0,884 & ncar_ccsm3_0_run6 & 40 & 0,767 & 0,637 & 0,848 & 0,751 \\
\hline cccma_cgcm3_1_t63_run1 & 4 & 0,906 & 0,845 & 0,892 & 0,881 & miroc3_2_medres_run1 & 41 & 0,818 & 0,627 & 0,802 & 0,749 \\
\hline giss_model_e_h_run1 & 5 & 0,976 & 0,775 & 0,891 & 0,881 & miub_echo_g_run5 & 42 & 0,669 & 0,740 & 0,828 & 0,745 \\
\hline ingv_echam4_run1 & 6 & 0,860 & 0,785 & 0,992 & 0,879 & miub_echo_g_run2 & 43 & 0,666 & 0,772 & 0,791 & 0,743 \\
\hline giss_model_e_r_run5 & 7 & 0,877 & 0,909 & 0,842 & 0,876 & bccr_bcm2_0_run1 & 44 & 0,835 & 0,795 & 0,596 & 0,742 \\
\hline giss_model_e_r_run2 & 8 & 0,879 & 0,890 & 0,816 & 0,862 & mri_cgcm2_3_2a_run3 & 45 & 0,708 & 0,759 & 0,756 & 0,741 \\
\hline giss_model_e_r_run8 & 9 & 0,877 & 0,874 & 0,830 & 0,860 & miroc3_2_hires_run1 & 46 & 0,904 & 0,402 & 0,912 & 0,739 \\
\hline giss_model_e_h_run2 & 10 & 0,972 & 0,676 & 0,898 & 0,849 & ncar_pcm1_run4 & 47 & 0,477 & 0,897 & 0,806 & 0,727 \\
\hline giss_model_e_r_run3 & 11 & 0,904 & 0,857 & 0,759 & 0,840 & miub_echo_g_run4 & 48 & 0,670 & 0,621 & 0,885 & 0,726 \\
\hline ipsl_cm4_run1 & 12 & 0,897 & 0,700 & 0,892 & 0,830 & ncar_pcm1_run1 & 49 & 0,482 & 0,893 & 0,801 & 0,725 \\
\hline giss_model_e_r_run4 & 13 & 0,879 & 0,800 & 0,798 & 0,826 & cccma_cgcm3_1_run2 & 50 & 0,835 & 0,551 & 0,789 & 0,725 \\
\hline giss_model_e_r_run7 & 14 & 0,878 & 0,847 & 0,727 & 0,817 & mpi_echam5_run1 & 51 & 0,827 & 0,571 & 0,777 & 0,725 \\
\hline giss_aom_run1 & 15 & 0,867 & 0,787 & 0,796 & 0,817 & giss_model_e_h_run5 & 52 & 0,981 & 0,447 & 0,743 & 0,724 \\
\hline giss_model_e_r_run6 & 16 & 0,879 & 0,647 & 0,916 & 0,814 & ncar_ccsm3_0_run5 & 53 & 0,761 & 0,680 & 0,721 & 0,721 \\
\hline giss_model_e_h_run3 & 17 & 0,844 & 0,743 & 0,843 & 0,810 & ncar_ccsm3_0_run3 & 54 & 0,666 & 0,684 & 0,806 & 0,719 \\
\hline giss_model_e_h_run4 & 18 & 0,977 & 0,740 & 0,702 & 0,806 & mpi_echam5_run2 & 55 & 0,840 & 0,555 & 0,756 & 0,717 \\
\hline mri_cgcm2_3_2a_run1 & 19 & 0,764 & 0,811 & 0,840 & 0,805 & giss_model_e_r_run9 & 56 & 0,886 & 0,559 & 0,702 & 0,716 \\
\hline miroc3_2_medres_run2 & 20 & 0,805 & 0,767 & 0,838 & 0,803 & csiro_mk3_5_run3 & 57 & 0,447 & 0,840 & 0,859 & 0,715 \\
\hline ncar_ccsm3_0_run4 & 21 & 0,762 & 0,843 & 0,793 & 0,799 & gfdl_cm2_0_run2 & 58 & 0,791 & 0,546 & 0,793 & 0,710 \\
\hline csiro_mk3_0_run3 & 22 & 0,605 & 0,890 & 0,877 & 0,791 & gfdl_cm2_0_run3 & 59 & 0,464 & 0,829 & 0,824 & 0,706 \\
\hline cccma_cgcm3_1_run5 & 23 & 0,840 & 0,922 & 0,588 & 0,783 & gfdl_cm2_1_run2 & 60 & 0,740 & 0,563 & 0,794 & 0,699 \\
\hline csiro_mk3_0_run2 & 24 & 0,941 & 0,780 & 0,623 & 0,782 & ncar_pcm1_run2 & 61 & 0,482 & 0,804 & 0,790 & 0,692 \\
\hline mri_cgcm2_3_2a_run4 & 25 & 0,767 & 0,761 & 0,810 & 0,780 & gfdl_cm2_1_run1 & 62 & 0,761 & 0,544 & 0,740 & 0,682 \\
\hline inmcm3_0_run1 & 26 & 0,923 & 0,646 & 0,758 & 0,776 & miub_echo_g_run1 & 63 & 0,681 & 0,580 & 0,755 & 0,672 \\
\hline ncar_ccsm3_0_run7 & 27 & 0,776 & 0,700 & 0,851 & 0,776 & mri_cgcm2_3_2a_run2 & 64 & 0,766 & 0,542 & 0,708 & 0,672 \\
\hline ncar_ccsm3_0_run9 & 28 & 0,762 & 0,817 & 0,734 & 0,771 & mpi_echam5_run3 & 65 & 0,638 & 0,625 & 0,708 & 0,657 \\
\hline cnrm_cm3_run1 & 29 & 0,906 & 0,550 & 0,852 & 0,769 & csiro_mk3_5_run2 & 66 & 0,679 & 0,529 & 0,755 & 0,654 \\
\hline iap_fgoals1_0_g_run3 & 30 & 0,955 & 0,533 & 0,812 & 0,767 & cccma_cgcm3_1_run3 & 67 & 0,622 & 0,873 & 0,425 & 0,640 \\
\hline gfdl_cm2_0_run1 & 31 & 0,788 & 0,775 & 0,731 & 0,765 & csiro_mk3_5_run1 & 68 & 0,676 & 0,456 & 0,762 & 0,631 \\
\hline ukmo_hadcm3_run1 & 32 & 0,915 & 0,531 & 0,835 & 0,760 & cccma_cgcm3_1_run4 & 69 & 0,837 & 0,721 & 0,335 & 0,631 \\
\hline ncar_ccsm3_0_run2 & 33 & 0,757 & 0,813 & 0,710 & 0,760 & miroc3_2_medres_run3 & 70 & 0,621 & 0,733 & 0,495 & 0,616 \\
\hline giss_aom_run2 & 34 & 0,870 & 0,629 & 0,777 & 0,759 & ncar_pcm1_run3 & 71 & 0,069 & 0,932 & 0,778 & 0,593 \\
\hline ukmo_hadcm3_run2 & 35 & 0,907 & 0,619 & 0,742 & 0,756 & iap_fgoals1_0_g_run1 & 72 & 0,730 & 0,153 & 0,688 & 0,523 \\
\hline mpi_echam5_run4 & 36 & 0,827 & 0,678 & 0,755 & 0,753 & iap_fgoals1_0_g_run2 & 73 & 0,726 & 0,131 & 0,664 & 0,507 \\
\hline ncar_ccsm3_0_run1 & 37 & 0,757 & 0,728 & 0,775 & 0,753 & & & & & & \\
\hline
\end{tabular}


Avaliação de Desempenho dos Modelos de Mudança Climática do IPCC-AR4 Quanto a Sazonalidade e os

Padrões de Variabilidade Interanual da Precipitação Sobre o Nordeste do Brasil, Bacia do Prata e Amazônia

GISS-AOM apresentaram correlações ligeiramente inferiores aos demais, dessa forma mostram-se inferiores aos outros modelos IPCC na representação da climatologia média sobre a região;

- Na bacia da Prata as rodadas dos modelos CSIRO_MK3_5, GFDL_CM2_0, GFDL_CM2_1, NCAR_CCSM_1_0 e IPSL_CM4 apresentaram $\mathrm{AVAL}_{\mathrm{s}}$ inferiores aos demais, por isso obtiveram uma classificação bastante baixa;

- Na região da Amazônia os modelos NCAR_CCSM_1_0, MIUB_ECHO_G e CSIRO_MK_3_5 apresentam correlações ou RMSE_PC ligeiramente inferiores aos demais modelos do IPCC na representação sazonal;

Quanto às avaliações interdecadal e interanual dos modelos IPCC algumas observações são destacadas:

- A maioria dos modelos capta os padrões de variação interanual, com algumas rodadas dos modelos globais do IPCC apresentaando valores bastante elevados de correlação em relação à precipitação observada durante o século XX. Porém, o mesmo não acontece com a avaliação interdecadal, a maioria dos modelos mostra muita dificuldade;

- As rodadas do modelo IAPG.FGOALS possuem dificuldade de representação plurianual na Amazônia e na Bacia da Prata;

- As rodadas dos modelos NCAR.CCSM.3.0, GFDL.CM.2.0 e GFDL.CM.2.1 possuem muita dificuldade de representar os padrões de variação no Nordeste setentrional;

A avaliação geral apontou como melhor modelo para o Nordeste setentrional o CSIRO.MK3.0.run2 seguido pelos modelos GISS.MODEL.E.R.RUN8 e UKMO.HADCM3.RUN1. Enquanto para Bacia da prata as rodadas do modelo canadense CCCMA.CGCM3.1 apresentaram altos valores de AVALg. Já para a Amazônia destacam-se as rodadas do modelo GISS.MODEL.E.R que apresentaram altos valores de AVALg. Estes modelos podem ser considerados como uma boa opção para avançar na investigação dos efeitos das mudanças climáticas sobre os recursos hídricos na América Sul.

\section{REFERÊNCIAS}

ALBURQUERQUE, I. F.; FERREIRA, J. N.; SILVA, M. G.;. DIAS, M. F. Tempo e Clima no Brasil. São Paulo: Oficina de Textos, 2009.

BOGGESS, A.; NARCOWICH, F. J. A first course in wavelets with fourier analysis. 2nd ed. Hoboken, New Jersey: John Wiley, 2009.

CAMPO, J. N. B.; NÉRIS, L. F. A. Mudanças climáticas e disponibilidades hídricas no semiárido: resultados preliminares. In: SERVAIN, J.; CAMPOS, J. N. B.; MARTINS, E. S. P. P. Clima do Atlântico Tropical e impactos sobre o Nordeste . 1. ed. Fortaleza: FUNCEME/IRD, 2010. v. 1.

COMPO, G. P.; WHITAKER, J. S.; SARDESHMUKH, P. D. Feasibility of a 100 year reanalysis using only surface pressure data. Bulletin of the American Meteorological Society, v. 87, p. 175-190, 2006.

IPCC. Intergovernmental Panel on Climate Change. Climate Change 2007: the physical science basis. Cambridge: Cambridge University Press, 2007a. 18 p.

IPCC. Intergovernmental Panel on Climate Change: Climate change 2007: impacts, adaptation and vulnerability. Cambridge: Cambridge University Press, 2007b. 23 p.

LÁZARO, Y. M. C. Avaliação dos modelos do IPCC AR4 quanto à sazonalidade e à variabilidade plurianual de precipitação no século XX em três regiões da América do Sul-projeções e tendência para o século XXI. 2011. $182 \mathrm{f}$. Dissertação (Mestrado). - Departamento de Engenharia Hidráulica e Ambiental, Universidade Federal do Ceará, Fortaleza, Ceará, 2011.

LORENZ, E. N A study of the predictability of a 28 Variable Atmospheric Model. Tellus, v. 17, n. 3, p. 321-333, 1965.

MARENGO, J. A.; SOARES, W. R. Impacto das mudanças climáticas no Brasil e Possiveis Cenários Climáticos: síntese do terceiro relatório do IPCC de 2001. Cachoeira Paulista, SP: CPTEC-INPE, 2005. p. 29.

MARENGO, J. A.; VALVERDE, M. C. Caracterização do clima no Século XX e Cenário de Mudanças de clima para o Brasil no Século XXI usando os mode- 
los do IPCC-AR4. Revista Multiciência Campinas, n. 8, maio 2007.

MILLY, P. C. D.; DUNNE, K. A.; VECCHIA, A. V. Global pattern of trends in streamflow e water availability in a changing climate. Nature, v. 438, n. 17, p. 347-350, 2005. doi:10.1038/nature 04312.

MOLION, L. C. B; BERNARDO, S. O. Uma revisão da dinâmica das chuvas no Nordeste Brasileiro. Revista Brasileira de Meteorologia, v. 17, n. 1, p. 1-10, 2002.

NEW, M.; HULME, M.; JONES, P. D. Representing twentieth century space-time climate variability. Part 1: development of a 1961-90 mean monthly terrestrial climatology. Journal of Climate, v. 12, p. 829-856, 1999.

NEW, M.; LISTER, D.; HULME, M.; MAKIN, I. A high-resolution data set of surface climate over global land areas. Climate Research, v. 21, n. 1, p. 1-25, 2001.

NOBRE, C. A. Vulnerabilidade, impactos e adaptação à mudança no clima. In: CADERNOS Núcleo de Assuntos Estratégicos da Presidência da República. № 3. Mudança do Clima. Vol I. Brasília: Núcleo de Assuntos Estratégicos da Presidência da República, Secretaria de Comunicação do Governo e Gestão Estratégica, 2005. v. 1, pt. 2, p. 147-216.

NOBRE, C. A.; SELLERS, P. J.; SHUKLA, J. Amazonian deforestation and regional climate change. Journal of Climate, v. 4, n. 10, p. 957-988, 1991.

NÓBREGA, M. T.; COLLISCHONN, W.; TUCGI, C. E. M.; PAZ, A. R. Uncertainty in climate change impacts on water resources in the Rio Grande Basin, Brazil. Hydrology and Earth System Sciences, v. 15, p. 585-595, 2011. Special Issue.

ROCHA, V. B. Uma abordagem de Wavelets aplicada à combinação de previsões: uma análise teórica $\mathrm{e}$ experimental. 155f. 2008. Dissertação (Mestrado em Métodos Numéricos em Engenharia) - Universidade Federal do Paraná, Curitiba, 2008.

RUCH, D.K.; FLEET, P. J.V. Wavelet theory: an elementary approach with applications. 2nd ed. Hoboken, New Jersey: John Wiley, 2009.

SALATI, T.; SCHINDLER, W.; VICTORIA, D. C.; SALATI, E.; SOUZA, J. C. S.; NOVA, N. A. V. Economia das Mudanças Climáticas no Brasil. Estimativas da
Oferta de Recursos Hídricos no Brasil em Cenários Futuros de Clima. Rio de Janeiro: Fundação Brasileira para o Desenvolvimento Sustentável, 2008. 80 p.

SILVEIRA, C. S.; SOUZA FILHO, F. A. Análise das projeções de precipitação do IPCC-AR4 para os cenários A1B, A2 E B1 para o século XXI para Nordeste Setentrional do Brasil. Revista Brasileira de Recursos Hidricos, 2011. Aceito com previsão de publicação no v. 18, n. 2, abril/junho de 2013.

SILVEIRA, C. S.; SOUZA FILHO, F. A.; LÁZARO, Y. M. C.; COSTA, A. C.; SALES, D. C.; COUTINHO, M. M. Sazonalidade da Precipitação Sobre o Nordeste Setentrional Brasileiro nas Simulações do IPCC-AR4. Revista Brasileira de Recursos Hídricos, v. 17, n. 3, p. 125-134, 2012.

SOUZA FILHO, F. A. Variabilidade e Mudança Climática nos Semi-Áridos Brasileiros. In: TUCCI, C. E. M.; BRAGA, B. (Org.). Clima e recursos hídricos no Brasil. Porto Alegre: ABRH, 2003. p. 77-111. (Coleção ABRH, v. 9).

SOUZA FILHO, F. A.; MOURA, A. D.Memórias do Seminário Natureza e Sociedade nos Semi-Áridos. Fortaleza: Banco do Nordeste do Brasil; Fundação Cearense de Meteorologia e Recursos Hídricos, 2006.

TEXEIRA, M.; SATYAMURTY, P. Dynamical and synoptic characteristics of heavy rainfall episodes in Southern Brazil. Monthly Weather Review, v. 135, n. 2, p. 598-617, 2007.

TOMASELLA, J.; RODRIGUEZ, D. A.; CUARTAS, L. A.; FERREIRA, M.; FERREIRA, J. C.; FERREIRA, J.C.; MARENGO, J. Estudo de impacto das mudanças climáticas sobre os recursos hídricos superficiais e sobre os níveis dos aqüiferos na Bacia do Rio Tocantins. Cachoeira Paulista, SP: CCST/INPE, 2009.

TORRENCE, C.; COMPO, G. P. A practical guide to wavelet analysis. Bulletin of the American Meteorological Society, v. 79, n. 1, p. 61-78, 1998.

UVO, G. R. B.; NOBRE, C. A. A Zona de Convergência Intertropical (ZCIT) e sua relação com a precipitação da região norte do Nordeste brasileiro. In: CONGRESSO INTERAMERICANO DE METEOROLOGIA, 2., 1987, Buenos Aires, AR., Anais... Buenos Aires, 1987. p. 6.9.1-6. 
WHITAKER, J. S.; COMPO, G. P.; WEI, X.; HAMILL, T. N. Reanalysis without radiosondes using ensemble data assimilation. Monthly Weather Review, v. 132, p. 1190-1200, 2004.

WILKS, D.S Statistical Methods in the Atmospheric Science: an introduction. San Diego: Academic Press, 1995.

Assessment Of The Performance Of The IPCC-AR4 Climate Change Models As To Seasonality And Patterns Of Interannual Variability Of Precipitation Over The Northeast Of Brazil, Plate Basin And Amazon Region

\section{ABSTRACT}

The global models of the fourth report of the Intergovernmental Panel on Climate Change (IPCC) are assessed for the Northeast of Brazil (NEB), the Plate Basin and the Amazon regarding the representation of precipitation for the 1901-1999 period. This assessment is performed using data from the Climatic Research Unit (CRU) and the 20th Century Reanalysis V2 of the National Oceanic and Atmospheric Administration (NOAA). The assessment consists of three steps that analyze patterns of variation at different scales: seasonal, interannual and interdecadal. The seasonal assessment is based on measures of correlation and mean square error in relation to the average climatology of the region, while the interannual and interdecadal assessments are based on wavelet transform. Most models capture the patterns of seasonal and interannual variation, but have great difficulty representing the interdecadal variability. The best models according to these criteria were in the Amazon region the GISS_MODEL_E_R model rounds; in the Plate Basin the CCCMA_CGCM3_1 model rounds.

Key-words: IPCC-AR4, South America and 20C3M scenarios. 